\title{
Report on the Second Annual Workshop on Naval Applications of Machine Learning
}

\author{
Katie Rainey, Josh Harguess
}

\begin{abstract}
The second annual workshop on Naval Applications of Machine Learning (NAML) was held February 13-15, 2018, at the Space and Naval Warfare (SPAWAR) Systems Center Pacific (SSC Pacific), a US Navy research laboratory in San Diego, California USA. The workshop events included invited speakers, demonstrations, discussion sessions, and oral and poster presentations. The workshop cochairs were Josh Harguess and Katie Rainey, both from SSC Pacific. The poster presentations were coordinated by Chris Ward, also from SSC Pacific. This article discusses the motivation, goals, and impact of the workshop and highlights some of the topics covered.
\end{abstract}

\begin{abstract}
$\mathrm{M}$ achine learning and artificial intelligence are rapidly accelerating fields, with estimated investments into AI startups alone of more than $\$ 6$ billion over the last three years (Agarwal et al. 2018). The importance of ML and AI with respect to national security has recently been underscored by the creation of the Joint AI Center (JAIC) (Freedberg 2018), whose goal is to have oversight over almost all service and defense agency AI efforts and to establish common "standards, tools, shared data, reusable technology, processes, and expertise" for the Department of Defense (DOD). The annual workshop on Naval Applications of Machine Learning (NAML) has been organized and held at Space and Naval Warfare (SPAWAR) Systems Center Pacific (SSC Pacific) twice in the past two years with goals similar to those of the JAIC and has been seen as a great success in at least starting the conversation in each of those goals. This article discusses the motivation, goals, and impact of NAML and highlights some of the topics covered.

SSC Pacific conducts research and development in support of integrated command, control, communications, intelligence, surveillance and reconnaissance (ISR), cyber, and space systems across all warfighting domains. These mission areas contain a multitude of applications for machine learning, and SSC Pacific's community of machine learning researchers has been actively growing for the past several years. SSC Pacific hosted the first NAML workshop in January 2017 with the intention of showcasing the work of the machine learning community to a mostly internal audience.
\end{abstract}


As interest grew, participation expanded to other Department of Defense-affiliated laboratories, academia, and industry. The workshop chairs were surprised and encouraged by the large variety of people supporting the US Navy in solving problems in these areas. The request for participation for the 2018 workshop was sent to a broad audience. Through improved publicity and more advanced planning (in addition to the explosion of interest in machine learning), attendance increased twofold from 2017, to nearly 400 people.

In this report, we give a recounting of the 2018 NAML workshop and its impacts. The next section gives a brief background and the motivation behind NAML. We then provide an overview of topics presented at the NAML workshop and discuss a few selected presentations in further detail. We end by providing the resulting impact of NAML and future plans for the event and conclude the report.

\section{Background and Motivation}

Interest in ML and $\mathrm{AI}$ is accelerating, and the establishment of the JAIC is the DOD's first step in officially recognizing the importance of this area and their commitment to building and maintaining the ML and AI communities within the department. Prior to the JAIC, the NAML workshop was one of only a handful of venues to obtain visibility into ML and AI projects within the US Navy and DOD, and no other venue has such a large and varied audience in attendance. Most projects in ML and AI within the DOD are very small, with common budgets from $\$ 100,000$ to $\$ 500,000$ per year and teams of only one to four people. Collaboration opportunities can help researchers on small projects maintain awareness of other related efforts to avoid duplicating work and to accelerate their progress. NAML provides a venue for sharing research ideas and results in ML and AI to the broader US Navy and DOD communities to provide support to researchers who most likely work on smaller projects with little to no DOD-wide visibility.

DOD research efforts are often shared at outside venues such as industry and academic conferences. But there are many unique requirements when applying machine learning to US Navy and DOD needs. Data availability and operating conditions, for example, may be significantly different than in industrial applications. The NAML workshop provides a forum within which to discuss approaches to some of these challenges with an audience closely connected to the warfighter. The goals of NAML are to spread awareness of current machine learning research relevant to navy applications, to connect machine learning researchers with experts in US Navy needs and requirements, and to build and strengthen collaborations within the DOD research community.

Most of the work presented was at the unclassi- fied, publicly releasable level; however, NAML also provides a unique venue for attendees to discuss their work at higher classification levels, which is very uncommon at other venues.

\section{The 2018 Workshop on Naval Applications of Machine Learning}

The NAML workshop welcomed participants from dozens of organizations within the DOD, including many of the laboratories in the Naval Research and Development Establishment (NR\&DE), as well as laboratories and commands under the US Army, US Air Force, US Marine Corps, and the US intelligence community. There were also attendees from industry (including traditional defense contractors and other companies), from universities and university-affiliated research centers, and from federally-funded research and development centers.

The workshop also featured three invited speakers. David Aha from the Naval Research Laboratory (NRL) gave a talk titled "Machine Learning in the Context of Goal Reasoning and Explainable AI." Guna Seetharaman, also from NRL, gave a talk titled "Computing Architectures: Post Moore's Law and AI/ML/DL Era." Travis Axtell from the Office of the Undersecretary of Defense for Intelligence gave a talk titled "AI Ignition," in which he discussed Project Maven, an effort that is providing computer vision algorithms for object detection, classification, and alerts in video and still imagery.

The main program consisted of short technical talks and poster presentations on a wide variety of topics related to machine learning. Most of the presenters shared current research efforts in support of US Navy or DOD programs, while some discussed strategic considerations of the US Navy with respect to machine learning or related issues. The oral presentations were organized into sessions on the topics of computer vision, autonomy, cognitive electronic warfare, algorithms and theory, and cybersecurity.

Two additional sessions showcased work by NR\&DE researchers on an assortment of topics. Thirty-five short talks were given, and over 50 posters presented, all selected from 110 abstract submissions.

The US Navy acquires data from a wide variety of sensors, presenting many opportunities for exploitation with machine learning. Several researchers presented their work investigating ways to analyze maintenance data to detect faults or to predict part lifecycles. Conventional machine learning methods are used on radar signals to identify behavior changes or to classify emitters. Scheduling algorithms are applied to ship data from US Navy ranges and to active sonar arrays. Several other presenters also discussed their work with yet other distinctive data types of interest to the US Navy, including infrasonic waves, marine geologic data, atmosphere aerosol data, and side-scan sonar. 
Other presentations were about work with data types that are common in work outside the DOD, but with US Navy-specific targets or goals. Some examples include detecting or tracking unmanned aerial vehicles in video, autonomous swarm tactics, sentiment analysis in social media data, classification of cognitive-motor interaction tasks in muscle and brain activity data, and network traffic classification.

Several presenters discussed military-relevant datasets, including a computer vision dataset collected in operational environments, and a set of threedimensional point clouds from US Navy ships. There was also a presentation on the DIUx xView 2018 Detection Challenge (Lam et al. 2018), including a publicly available satellite imagery dataset released by the Defense Innovation Unit Experimental (DIUx) and the National Geospatial-Intelligence Agency (NGA).

The US Navy is interested in fundamental questions about the capabilities and limitations of machine learning. Several presentations discussed basic and applied research efforts and the ways in which that research advances US Navy goals. Some of the work presented was performed by students supporting the Naval Research Enterprise Internship Program (NREIP) or the Science, Mathematics and Research for Transformation (SMART) Scholarship for Service Program. There were also presentations from some of the US Navy's academic and industrial partners.

On the final afternoon of the workshop, several tutorials, demonstrations, and discussion sessions were held in two concurrent tracks. Justin Mauger and Benjamin Migliori of SSC Pacific gave a tutorial on topological data analysis and spiking neural nets. Kristin Lauter of Microsoft Research gave a tutorial on homomorphic encryption. Tom Schlosser and Joe Drobick of SSC Pacific gave a demonstration of a suite of US Navy collaboration tools. Chris Ward of SSC Pacific demonstrated recent work on microelectronics characterization and analysis. Informal discussion sessions, intended to enable attendees to meet one another and establish collaborations, were held on the topics of autonomy, biologically inspired machine learning, computer vision, cognitive electronic warfare, cybersecurity, and predictive analytics. While several discussion sessions this afternoon were open only to restricted audiences, the rest of the workshop was held at the unclassified level and was open to all attendees.

\section{Selected Presentations}

Although it's not possible to cover every presentation in this summary report, what follows is a sampling of selected presentations given at NAML 2018. A complete listing of presentations, including most of the presented abstracts, will be published in a technical report that will be publicly available on the Defense Technical Information Center (DTIC). ${ }^{1}$
Dean Lee of SSC Pacific presented "Applications of Machine Learning with V-22 Operational Data." In his work, Lee applies machine learning and data mining to predictive maintenance for the $\mathrm{V}-22$ program (Lee et al. 2016).

Jennifer Williams of the Naval Undersea Warfare Center (NUWC) Keyport Division and Emily Nystrom of SPAWAR Systems Center Atlantic presented their work on obsolescence management in talks titled "Applying Machine Learning and Data Mining to Obsolescence Management" and "Applications of Random Forests for Modeling Obsolescence," respectively.

Andrew Christianson of the Naval Surface Warfare Center (NSWC) Crane Division presented "Classification of Radar Signals" (Kaiser, Christianson, and Narayanan 2016), while Diego Marez of SSC Pacific presented "Electronic Warfare Activity Recognition," summarizing his recent work.

Work by Sandy Kelly of NUWC Keyport Division and Thomas Powers of the University of Washington looked at optimization and scheduling for US Navy missions in two presentations titled "Support Vessel Scheduling for Pacific Northwest Test Ranges" and "Avoiding Catastrophes: Worst-Case Optimization with Applications to Multistatic Active Sonar Arrays."

Finally, two presenters focused on undersea applications of machine learning. Warren Wood of the Naval Research Laboratory (NRL) presented "Machine Learning Prediction of Seafloor Properties" (Martin, Wood, and Becker 2015), and Daniel Gebhardt of SSC Pacific presented "Hunting for Naval Mines with Deep Neural Networks" (Gebhardt et al. 2017).

\section{Impact and Future Plans}

The impacts of NAML are severalfold. First, several new collaborations between US Navy and DOD researchers were formed. One such collaboration involves data collection for a small robotic platform dataset in real-world degraded environments between SSC Pacific, NRL, and the Army Research Laboratory (ARL). This collaboration will directly support a research effort at SSC Pacific to better understand the effects of real-world data on motion estimation from imagery (Harguess, Barngrover, and Rahimi 2017; Harguess, Marez, and Ronquillo 2018). Another new collaboration was a research proposal on topological data analysis between mathematicians at SSC Pacific and NRL. Additionally, to meet the demands of data-starved machine learning algorithms and the lack of relevant annotated data, a collaboration between NR\&DE laboratories was formed to build and maintain a data repository for storing and sharing large datasets between the NR\&DE and the larger US Navy and DOD communities. Second, the meeting satisfied its main motivation, which was to provide a venue for US Navy and DOD researchers 
to present and participate in discussions on the applications of machine learning to relevant problems and datasets. While there are other venues to present this type of work, NAML's audience is unique due to the large attendance by US Navy and DOD researchers in contrast to the mostly academic or industry attendees of other workshops. The third and possibly most important impact of NAML is the establishment of a grassroots-driven community of interest around the topics of machine learning, computer vision, and artificial intelligence. It is our intention to continue holding the NAML workshop annually at SSC Pacific, although with a few potential changes and additions. First, due to the popularity and limited space for the event, we will steer the workshop towards specific technology and problem areas for the US Navy and accept abstracts in those areas instead of opening the workshop to any application of machine learning. For instance, there was a lot of interest in the use of machine learning on cyber problems, so we will plan to hold a session focused on that area. Second, we plan to hold smaller, more focused workshops whose goal is to tackle, or at least make progress on, a specific problem within the US Navy. We hope to have researchers from other US Navy labs and organizations lead these workshops and report back their findings to the larger NAML audience each year. The third annual NAML workshop is scheduled for 11-14 February at SSC Pacific. More information about the workshop is available at the workshop website. ${ }^{2}$

\section{Conclusion}

SSC Pacific organized and ran the second annual workshop on Naval Applications of Machine Learning (NAML) on February 13-15, 2018. The event was well attended and was largely considered a great success. This paper is an attempt to summarize the event, impact, and future plans. The event spurred many new, and fostered many continuing, collaborations. We plan to continue organizing the workshop annually and to continue holding it at SSC Pacific every winter, with the addition of specialized and more focused workshops to be held between the annual events. Events such as this are crucial to the communication of ideas, problems, and solutions between researchers in labs around the US Navy and DOD and other organizations.

\section{Notes}

1. www.dtic.mil/.

2. sites.google.com/go.spawar.navy.mil/naml/.

\section{References}

Agarwal, D.; Bersin, J.; Lahiri, G.; Schwartz, J.; and Volini, E. 2018. AI, Robotics, and Automation: Put Humans in the Loop. Deloitte Insights, March 28. www2.deloitte.com/ insights/us/en/focus/human-capital-trends/2018/ai-robotics-intelligent-machines.html.

Freedberg, S. J. Jr. 2018. Joint Artificial Intelligence Center Created Under DoD CIO. Breaking Defense. June 29. breakingdefense.com/2018/06/joint-artificial-intelligence-centercreated-under-dod-cio.

Gebhardt, D.; Parikh, K.; Dzieciuch, I.; Walton, M.; and Hoang, N. A. V. 2017. Hunting for Naval Mines with Deep Neural Networks. In OCEANS 2017-Anchorage, 1-5. Piscataway, NJ: Institute for Electrical and Electronics Engineers.

Harguess, J.; Barngrover, C.; and Rahimi, A. 2017. An Analysis of Optical Flow on Real and Simulated Data with Degradations. In Proceedings of the International Society for Optics and Photonics 10199: Geospatial Informatics, Fusion, and Motion Video Analytics VII, 1019905. Bellingham, WA: SPIE. doi.org/10.1117/12.2265850.

Harguess, J.; Marez, D.; and Ronquillo, N. 2018. An Investigation into Strategies to Improve Optical Flow on Degraded Data. In Proceedings of the International Society for Optics and Photonics 10645: Geospatial Informatics, Motion Imagery, and Network Analytics VIII, 106450F. Bellingham, WA: SPIE. doi.org/10.1117/12.2305295.

Kaiser, S. A.; Christianson, A. J.; and Narayanan, R. M. 2016. Multistatic Radar Exploitation of Forward Scattering Nulls. In Proceedings of the 2016 IEEE Radar Conference, 1-6. Piscataway, NJ: Institute for Electrical and Electronics Engineers.

Lam, D.; Kuzma, R.; McGee, K.; Dooley, S.; Laielli, M.; Klaric, M.; Bulatov, Y.; and McCord, B. 2018. xview: Objects in Context in Overhead Imagery. arXiv preprint. arXiv:1802.07856 [cs.CV]. Ithaca, NY: Cornell University Library.

Lee, D.; Siu, V.; Cruz, R.; and Yetman, C. 2016. Convolutional Neural Net and Bearing Fault Analysis. In Proceedings of the International Conference on Data Mining, 194-200. Athens, GA: CSREA Press.

Martin, K. M.; Wood, W. T.; and Becker, J. J. 2015. A Global Prediction of Seafloor Sediment Porosity Using Machine Learning. Geophysical Research Letters, 42(24). doi.org/ 10.1002/2015GL065279.

Katie Rainey is a scientist at Space and Naval Warfare Systems Center Pacific, a U.S. Navy research laboratory in San Diego, California. Her research interests include object recognition in the maritime domain and $\mathrm{AI}$ robustness. She also works to direct research to support defense applications, and is active in building the community of AI researchers among defense laboratories. She has a $\mathrm{PhD}$ in mathematics from the University of California, Irvine.

Josh Harguess has been with Space and Naval Warfare Systems Center Pacific for six years focusing on research in computer vision and machine learning applied to applications of interest to the Navy and DoD, such as ship detection and tracking, video content analysis, 3D model generation, and Augmented Reality. He is an active researcher in these areas with more than 45 publications and 5 patents. He received his PhD from the University of Texas at Austin working on face recognition from multiple video cameras. 